\title{
Toward Preventing Cascading Blackouts: Vulnerability and Criticality Stress Metrics
}

\author{
Md Abid Hossain \\ University of Utah \\ abidzim08@gmail.com \\ Donald G. Davies \\ Marc Bodson \\ Western Electric Coordinating Council University of Utah \\ ddavies@wecc.org \\ marc.bodson@utah.edu \\ Hyde M. Merrill \\ University of Utah \\ hyde@merrillenergy.com
}

\begin{abstract}
A simple, effective approach is presented for measuring the susceptibility of power systems to cascading blackouts. A failure network and an analysis method are proposed, based on line outage distribution factors and network theory. This network is analyzed with metrics that quantify stress. The metrics can be computed rapidly and be used in realtime operations as well as for. The studies presented are remarkable for use of real, large-scale data for the entire Western Interconnection of North America. The metrics vary reasonably with different seasonal and loading conditions. Metrics for a pre-blackout reconstruction show that the system was highly stressed and correctly identified the most vulnerable and critical areas and branches. Lowering stress is a serious candidate for reducing risk of cascading.
\end{abstract}

\section{Introduction}

Cascading blackouts are the oldest major unsolved technical problem in power system engineering, since before a famous 1965 event. Two generations of engineers have struggled with this puzzling and vexing problem. Undamaged systems fail, but not from external causes. Cause-and-effect cascades affect large areas and are difficult to recover from.

The power system is a force-at-a-distance, humanmachine energy conversion system of three elements: (1) hardware to generate and transmit current, (2) control and protection equipment, and (3) practices and procedures.

The second and third elements are too complex to be error-proof. They must be designed and operated with care, but perfect performance is not practical.

This paper considers the problem of cascading, where one failure causes others in a chain, in an essentially undamaged bulk power system. History reveals that cascading blackouts occur when failures arise in the second or third elements in the above list, when large systems are stressed.
Simple, effective metrics based on network and power system theory measure stress, the susceptibility of a system to cascading failures. A new network, based on known engineering principles, models how failures flow through a system. In contrast, traditional networks model how power flows through a system. We show that modelling or simulating all of the phenomena of cascading blackouts is not required.

The stress metrics are computed for very large (WI, the Western Interconnection of North America) and large (SW WI, southwestern WI) systems in different conditions. The results match ad hoc stress reasonability judgments. Metrics for a pre-blackout state show that the overall stress was very high, particularly in cascading areas.

\section{Background}

A cascading blackout is an uncontrolled, unexpected chain of cause-and-effect failures in hardware for generating and carrying current. It interrupts bulk power service over a large area. Cascading blackouts are of concern because it can take hours or days to restore the system. Meanwhile, many functions of society are disrupted, sometimes with economic losses of billions of US dollars.

\subsection{Typical cascading blackouts}

The 9 November 1965 Northeast blackout occurred with relatively heavy flows on five essentially-parallel $230-\mathrm{kV}$ lines from the Niagara Falls area to Toronto, Ontario, Canada. Unbeknownst to the operators, backup relays were set far below the lines' true ratings, procedural failures. A relay tripped one line. Flows on the others increased. They tripped, one by one, separating most of the Ontario system from New York. Ontario had too much load and New York and nearby systems had too much generation. Neither could absorb the sudden imbalances. Both 
broke up into islands and blacked out [1].

The 14 August 2003 blackout cost the US from US $\$ 4$ billion to US $\$ 10$ billion. Canada's GDP was reduced. Procedural and computer failures in two control centers led to it. Three $345-\mathrm{kV}$ lines failed between 15:05 and 15:46 EDT. Not overloaded, they sagged into trees that were taller than they should have been. The post-mortem concluded that at "15:05 EDT ... the system was electrically secure [2]." This apparently was based on n-1 analyses with key line ratings that were much too high, a procedural failure. Expecting perfection in practices and procedures is neither useful nor an acceptable excuse. The system has to survive inevitable errors.

In 41 minutes, at 15:46, "the blackout might have been averted [but] it may already have been too late [2]." The final straw, tripping a fourth $345-\mathrm{kV}$ line at $16: 06$, was due to a relay interpreting high current and low voltage as a short circuit.

At 3:00 a.m. on Sunday 28 September 2003 (offpeak time, day, and month), Italy was importing 300 MW more than its scheduled imports from France, mainly via Switzerland, charging its pumped hydro plants. A $380-\mathrm{kV}$ Swiss line heated, sagged into a tree, flashed, and stayed open. The Swiss asked the Italians to reduce imports by $300 \mathrm{MW}$ to meet the schedule, which assumed all lines were in service. They could have asked, but did not ask the Italians to stop pumping. Another line tripped at 3:25 a.m. In seconds all lines into Italy tripped, and in three minutes all mainland Italy was blacked out [3] [4].

\subsection{Cascading blackouts that did not occur}

On 11 April 1965, 37 "Palm Sunday" tornados in and near Ohio took 27 American Electric Power Co. transmission lines and two extra-high voltage (EHV) substations out of service. Customers on failed radial lines lost power, but no cascading was reported [5]. It was Sunday in an off-peak month. The system was lightly loaded - unstressed by any definition. (It was not designed to survive 29 contingencies.)

Other large-system, non-blackout events have been discovered. Smaller systems seem not to cascade [6], e.g., the Texas system advised one author that it has been in extremis several times without cascading [7] [8].

\subsection{Other large-scale blackouts: resilience}

Puerto Rico was blacked out due to hurricanes Irma (service lost by two-thirds of electric customers) and Maria (the electrical infrastructure totally destroyed) in September 2017. In the January 1998 Quebec - New York ice storm, every overhead line (transmission and distribution) in a large area was on the ground. There was a blackout, but no cascading.

Significant work is being done on a problem complementary to ours, blackouts due to major disasters, in part developing the notion of resilience [9] [10]. There are three key differences between such events and the cascading failures of this paper. (1) These major disasters do not always result in cascading. The 1965 Palm Sunday tornadoes and the 1998 ice storm [11] caused great destruction but no cascading. (2) They cause extensive physical damage to the system, especially to the distribution system, which can take weeks or months to repair. Cascading failures mostly involve the bulk system, with little or no physical damage. (3) Causes of non-cascading blackouts include disasters (hurricanes, earthquakes [9] [10], cyber-attacks [12]), while cascading events are due to internal failures. The two problems have different causes, effects, and solutions.

For both of these problems, traditional reliability does not go far enough. They also have in common the definition and application of metrics. These metrics evaluate resilience (to extreme external events) or stress (for cascading). The two sets of metrics and their application are very different [9].

\subsection{Cascading failures: previous work}

The U.S.-Canadian power industry created NERC (now the North American Electric Reliability Corp.) after the 1965 blackout to improve reliability. Its planning and operating criteria have evolved in detail while being stable in basic concepts. NERC " $n-1$ " and other standards generally assume that control and protective devices, and policies and procedures, will work properly, with limited exceptions. Preventing cascading always has been central [13].

State estimation was introduced to provide accurate inputs to real-time procedures for increasing reliability [14].

A NERC study found that 73.5 percent of significant events were aggravated by "hidden failures" of the protection system, part of the second power system element above [15], [16].

Years ago it was observed that "Power systems have grown enormously and have become interconnected over vast regions. And we have had two severe blackouts and are undoubtedly headed for more." Also, "The more complex a society, the more chance there is that it will get fouled up [17]." 
About one-sixth of an excellent 1978 paper on the future power system was on blackouts. It concluded, "[Cascading] blackouts will not exist in the year 2000 ... There is a good chance that by the year 2000 the term [cascading blackout] will be considered to be a term out of the Dark Ages (sic) [18]."

Much labor has been invested on the cascading problem [19]. But misconceptions can lead to questionable conclusions. For example, "Since power systems are generally operated to be $\mathrm{N}-1$ secure, most historical cascades have been triggered by multiple [independent] outages in combination, motivating the need for probabilistic analysis [19]."

Multiple independent outages have caused few, if any, cascades, so this conclusion is unsupported. In all cascading blackouts known to the authors, system operation clearly was not NERC compliant (though operators might have thought that it was): a single outage caused cascading, a criteria violation.

\section{5. $\mathrm{N}-1$ criteria are not sufficient}

The post-mortem report of the 14 August 2003 US/Canada blackout concluded, "Although FirstEnergy's system was technically [i.e., by n-1 standards] in secure electrical condition before 15:05 EDT, it was still highly vulnerable [2]." (Italics added by authors)

This is authoritative recognition that current standards are not sufficient for preventing cascading.

A recent paper called the 1965 Northeast blackout an n-5 event, and systems cannot be designed to withstand these. The implication: occasional blackouts are inevitable [1]. With respect, the authors believe that the 1965 outage was not an n-5 event. The tripping of the first line (n-1) caused the other four to trip in sequence (n-1-1-etc.). The distinction is important. Such events are not inevitable.

\section{A cascading outage network}

\subsection{Power flow network}

Fig. 1 is the basic model of a network. The oneline (positive sequence) network is the basis for most power system modeling of the bulk transmission network. This model uses the Ybus (bus admittance) matrix. (Bold type indicates vectors and matrices.)

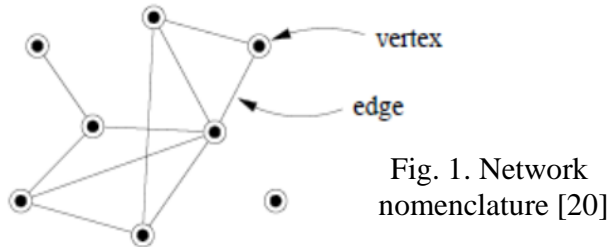

The Ybus network has the following properties:

- Ybus is square and symmetric.

- Each row and column is for a bus (vertex).

- Each edge is a branch (line or transformer).

The off-diagonal terms are branch admittances between two buses. Most are zero, since each bus is connected to few others. So Ybus is very sparse. The laws of Ohm and Kirchhoff determine how demand and generation affect bus voltages and branch flows.

\subsection{Cascading failure network}

The cascading outage problem needs a different model since the objective is to find how the outage of one branch affects the flows on other branches. In the cascading network, the vertices are branches (lines, transformers, interfaces), not buses. Each branch is linked to every other branch, not by single lines, but by the network as a whole. Edges in a cascading network are modeled by Line Outage Distribution Factors (LODF) or simply Distribution Factors (DFAX), defined by:

$$
\text { flow }_{k j}=\text { flow }_{k 0}+\left(D F A X_{k j} \times \text { flow }_{j 0}\right) .
$$

In (1), flow flo $_{0}$ and flow $_{k j}$ are the pre- and postoutage flows in monitored branch (k), and flow flo $_{0}$ is the pre-outage flow in outaged branch (j). The postoutage flow in branch (j) is zero, and $-1 \leq D F A X_{k j} \leq$ +1 .

- DFAX, containing the elements $D F A X_{k, j}$, with $\mathrm{m}_{\mathrm{o}}$ rows and $\mathrm{m}_{\mathrm{m}}$ columns, is much larger than YBUS. The subscripts "o" and " $m$ " refer to "outaged" and "monitored".

- Each row is for an n-1 outage branch.

- Each column is for a branch whose flow is monitored (computed) for each n-1outage.

Commercial power-flow programs compute the elements of DFAX from Ybus using the laws of Ohm and Kirchhoff. Branch limits are based on such effects as tree heights in rights-of-way. Two electrically identical lines in different rights-of-way may experience the same n-1 flow after a contingency. One may overload but the other not, due to different limits. Therefore, failure propagation through the DFAX network is complicated in a different way than is power flow.

\subsection{Typical DFAX matrices}

Fig. 2 shows the distributions of the absolute values of millions of DFAX for southwestern WI 
(SW WI) for three years: 2016 and 2012 (summer high demand), and 8 Sept. 2011 Pre-blackout. Other systems have similar curves [6]. Plotted on log-log scale, each density function (not shown) is close to a straight line, a power law, common in large networks.

In Fig. 2, each point $x, y$ on a curve is the number ' $y$ ' of DFAX whose absolute value is greater than or equal to the number ' $\mathrm{x}$ '. (Neither this figure, nor this paper, expresses or deals with probabilities.)

Even in a five-year interval with relatively low demand growth, the system evolved to have $30 \%$ more higher-value DFAX $(\geq 0.7)$. It became more tightly coupled and more susceptible to cascading.

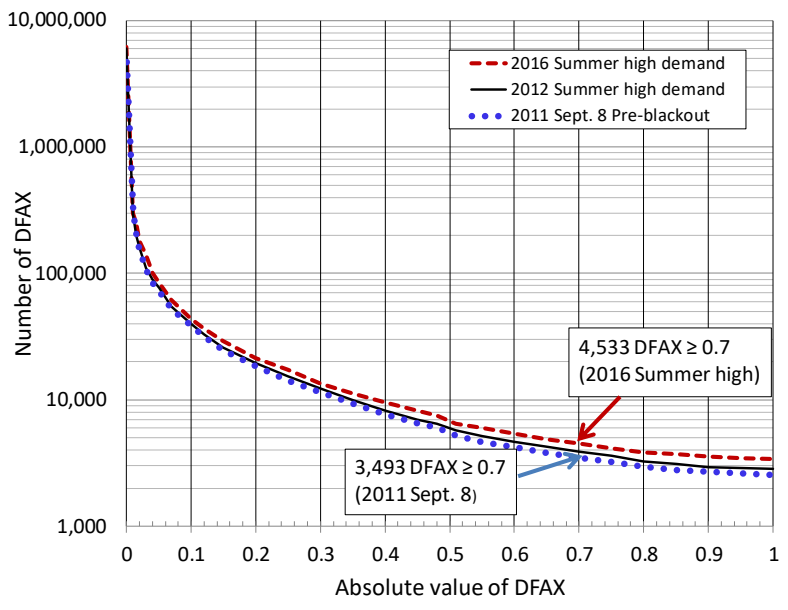

Fig. 2. DFAX distributions for SW WI.

\section{Stress metrics}

\subsection{Previous applications of network theory}

Euler's 1736 solution of the Konigsberg bridge problem is considered the beginning of network or graph theory, but most of its development has occurred in the past 100 years.

For our purposes, network theory responds to three observations.

- $\quad$ Large networks are pervasive.

- They are too big to study element by element.

- "Big is different." "Large" (not precisely defined) networks behave differently than small networks.

"The problem with systems like the power grid is that they are built of many components whose individual behavior is ... well understood ... but whose collective behavior ... can be sometimes orderly and sometimes chaotic, confusing, and even destructive [21]."

Therefore, concludes network theory, for large networks one must study structural properties and metrics. References [21] and [20] survey network theory and give many examples.

Network theory has been applied to a variety of problems in power, including attempting to determine contributions of each generator to each load [22] and generating random networks for studying widespread failures [23].

Network theory applications to cascading blackouts always use models where buses are vertices and lines are edges [24], [25], [26], [27], [28]. They consider a random failure at one bus propagating to a neighboring bus. They usually are based on abstract networks rather than real power systems. They do not seem to use Ohm's and Kirchhoff's laws to model the mechanisms for failure propagation.

A number of researchers have done work that resonates strongly with the approach of this paper. Three notable papers are mentioned here. The first [15] argues persuasively that the cascading problem is neither well formulated nor well addressed. The "present practice in system planning and on-line security analysis neglects the impact of the protection system." Furthermore, relying on n-1 criteria is inadequate, and the "probability of cascading failures is much higher than the probability of random (i.e. independent) tripping of $\mathrm{k}$ out of $\mathrm{N}$ components of the system."

A second study [16] identifies and simulates problems which affect cascading: network loading, spinning reserve, controls, and "hidden failures" (limited to the protection system). Hidden failures are not manifested until the system is stressed. Failure dynamics are modelled probabilistically. Probabilities increase from $\mathrm{Pr}=0$ below a threshold loading (for instance, 100 percent of rating) to $\mathrm{Pr}=1$ above a second threshold (say, 140 percent of rating).

An EPRI project [29] analyzed $n-k$ conditions on a 3 -area part of the 50,000-bus and 65,000-branch Eastern Interconnection of North America. Analyzing all $n-k$ conditions was impossible. A few $(>31,000)$ independent $\mathrm{n}-2$ outage combinations were created and tested from a user-supplied list of perhaps 250 single contingencies. Most $\mathrm{n}-2$ events ended uneventfully after overloaded elements tripped. Some 38 led to voltage collapse or islanding, but none took more than three steps to do so. Particularly intriguing are the small number of $n-2$ outages that resulted in overloads, and the huge fraction of these overloads that did not lead to further cascading.

Next we introduce two key concepts as bases of this paper: when the system is stressed, it is more susceptible to cascading from one of many improbable failures, most of which lack models or data. Therefore, our focus is on modeling and measuring stress to measure risk of cascading. 


\subsection{Vulnerability metrics}

Vulnerability is assessed from the $n-1$ flow in a branch after the outage of other branches in the system. This is a reasonable measure of stress, as cascading always begins with one outage causing one or more branches to become highly loaded and fail.

Vulnerability rank is the maximum absolute value of n-1 flow through a branch. Rank $\mathbf{V}$ is an $m_{m} \times 1$ column vector. Rank $V_{k}$ is the maximum post-outage flow on monitored branch $(k)$ for the outage of all $m_{j}$ outage branches, taken one at a time. $\operatorname{Rank}_{\mathrm{k}}$ may be greater than, less than, or equal to the precontingency flow on branch (k). See (1) and (2).

$$
\operatorname{Rank}_{k}=\max \left(\frac{\mid \text { flow }_{k, j} \mid}{\text { rating }_{k}}\right) \text { for all outages }(j)
$$

Vulnerability degree is the number of outages for which a monitored vertex will be heavily loaded. This definition differs slightly from the common usage of degree. In our examples "heavily loaded" means a threshold of 75 percent or 100 percent of rating. Specific thresholds will be discussed later. DegreeV is an $m_{m} \times 1$ vector. Degree $V_{k}$ is the number of outages for which the absolute value of the $\mathrm{n}-1$ power flow on vertex $(\mathrm{k})$ equals or exceeds its threshold. See (3), (4).

$$
\begin{gathered}
\text { DegreeV }_{k}=\sum_{j=1}^{m_{o}} \text { HighrankV }_{k, j} \text { where } \\
\text { HighRankV }_{k, j}= \\
\begin{array}{c}
\text { if }\left(\frac{\mid \text { flow }_{k, j} \mid}{\text { rating }_{k}}<\text { Threshold }_{j}\right), \\
\text { else }=1 .
\end{array}
\end{gathered}
$$

\subsection{Criticality metrics}

The criticality of a vertex assesses the seriousness of post-outage (n-1) flows in the system following its outage.

Criticality rank, $\operatorname{RankC}$, is a $1 \times \mathrm{m}_{\mathrm{o}}$ row vector. $\operatorname{RankC}_{\mathrm{j}}$ is the maximum n-1 flow, as a fraction of monitored branch rating, over all monitored vertices after the outage of vertex (j) (5).

$$
\begin{aligned}
& \text { RankC }_{j}=\max \left(\frac{\mid \text { flow }_{k, j} \mid}{\text { rating }_{k}}\right), \\
& \text { over all monitored vertices }(k) .
\end{aligned}
$$

Criticality degree, DegreeC, is a $1 \mathrm{x} \mathrm{m}_{\mathrm{o}}$ row vector. Degree $C_{j}$ is the number of monitored vertices (k) whose flows will equal or exceed their thresholds after the outage of vertex (j) (6), (4).

$$
\text { DegreeC }_{j}=\sum_{k=1}^{m_{m}} \text { HighrankV }_{k, j} .
$$

\subsection{Clarifications and illustration}

A mnemonic may be helpful: a vulnerable branch is a victim. A critical branch is a culprit. These definitions are for single branches. For a system, the metrics are distributions (which are not probabilities).

Equations (5) and (6) are very much like (2) and (3). They differ in the subscripts. See Table I, which is useful for understanding this paper. Vulnerability analyses are done by rows, criticality by columns.

The n-1 flows for three monitored vertices (branches), the heart of the table, are show in yellow, for two outaged vertices that are part of a larger system. Branch 2 is a path or interface of series/parallel branches, monitored but not outaged.

Vulnerability Computation (RankV, DegreeV, tan area): $\operatorname{RankV} V_{2}=\max n-1$ flow on branch2 $=|-1.39|$

\begin{tabular}{|c|c|c|c|c|}
\hline & \multicolumn{3}{|c|}{ Criticality (outaged vertices "j") } \\
\hline & & RankC & 2.43 & 0.87 \\
\hline & & DegreeC* & 2 & 1 \\
\hline \multirow{2}{*}{$\begin{array}{c}\text { RankV } \\
0.49\end{array}$} & DegreeV* & vertex & 1 & 3 \\
\hline & 0 & 1 & 0 & 0.49 \\
\hline \multirow{2}{*}{$\begin{array}{l}1.39 \\
2.43\end{array}$} & 2 & 2 & -1.39 & 0.87 \\
\hline & 1 & 3 & -2.43 & 0 \\
\hline \multicolumn{2}{|c|}{$\begin{array}{c}\text { Vulnerability } \\
\text { (monitored vertices "k") }\end{array}$} & & \multicolumn{2}{|c|}{$\begin{array}{l}\text { n-1 flows (fractions } \\
\text { of monitored } \\
\text { vertex ratings) }\end{array}$} \\
\hline
\end{tabular}
of the rating of branch2. The max is for the outage of branch1. Degree $V_{2}=2$ because the outage of either of two vertices ( 1 or 3 ) would cause |flow2| to exceed the chosen threshold of $75 \%$ of the rating of branch 2 .

TABLE I

Vulnerability and criticality metrics illustrated

* Thresholds $=75 \%$ of monitored vertex ratings

Criticality computation (RankC, DegreeC, blue area): $\operatorname{RankC}_{1}=$ maximum $\mathrm{n}-1$ flow of any branch for the outage of vertex $1=|-2.43|$. DegreeC $C_{1}=2$ as two monitored vertices have $\mid n-1$ flow $\mid>0.75$ for the outage of vertex 1 .

Interpreting Table I: RankC and DegreeC agree vertex 1 is more critical than vertex 3 . RankV and DegreeV agree - Vertex 1 is the least vulnerable.

\section{Diagnosing stress using metrics}

The system analyzed below evolves constantly as facilities are added and upgraded. Operating states change from constantly. Results from past data may not reflect the state of the system at any other time. 


\subsection{Western Interconnection study}

Analyses of stress for the eastern US and Peru have been presented [30] [6] [31]. Here, we report indepth studies of North America's Western Interconnection (WI) expanding on the preliminary results of [32].

The study of the WI used high quality power flow base cases (see Table II). All were built by WI utilities and WECC and are realistic and consistent. Base case access is limited. Data and results are tightly controlled under CEII (Critical Energy Industry Infrastructure) or similar rules. It is unheard of to give researchers access to pre-blackout cases.

TABLE II

Western Interconnection cases studied

(Statistics below are for the Southwestern region only)

\begin{tabular}{ccc}
$\begin{array}{c}\text { Power Flow Case } \\
\text { (Season, Demand) }\end{array}$ & $\begin{array}{c}\text { Demand / } \\
\text { Generation } \\
\text { (MW) }\end{array}$ & $\begin{array}{c}\text { Monitored } \\
\text { Vertices }\end{array}$ \\
\hline 2016 Summer High (HS) & $62,691 / 57,578$ & 2,419 \\
2016 Spring High (HSP) & $44,229 / 40,472$ & 2,456 \\
\hline 2016 Winter High (HW) & $38,931 / 36,085$ & 2,474 \\
2016 Winter Low (LW) & $27,530 / 30,500$ & 2,462 \\
\hline 2016 Summer Low (LS) & $34,577 / 32,010$ & 2,401 \\
2012 Summer High & $61,933 / 57,841$ & 2,215 \\
\hline 8 Sept. 2011 Pre-blackout & $51,619 / 46,752$ & 2,088 \\
\hline
\end{tabular}

Most analyses below consider only vertices (lines, transformers) in SW WI, but the DFAX reflected the entire WI. Normal system changes caused minor variations in the number of branches.

\subsection{Stress metrics and operating states}

Stress metrics for individual branches are scalars. Stress metrics for systems are distributions of branch metrics. As mentioned earlier, distributions should not be confused with probabilities. Fig. 3 shows system vulnerability metrics for several cases. Most vertices have RankV $<1.0$, consistent with the $\mathrm{n}-1$ criteria.

Up to 295 vertices (12 percent of the 2,400+ vertices) have RankV equal to or greater than the branch ratings, mainly in high-stress seasons, High Demand Summer and Spring. For three seasons, up to 20 vertices have $\mathrm{n}-1$ flows more than twice their ratings. These may be NERC $n-1$ violations.

One might ask how a base case can violate the $\mathrm{n}-1$ criteria. The base cases are hypothetical, made for WI operations planning studies. They are used to identify possible problems and do not include "fixes" for all of them. For example, a lower-voltage branch may be ignored, based on the assumption that events in the lower voltage system will not cause cascading.

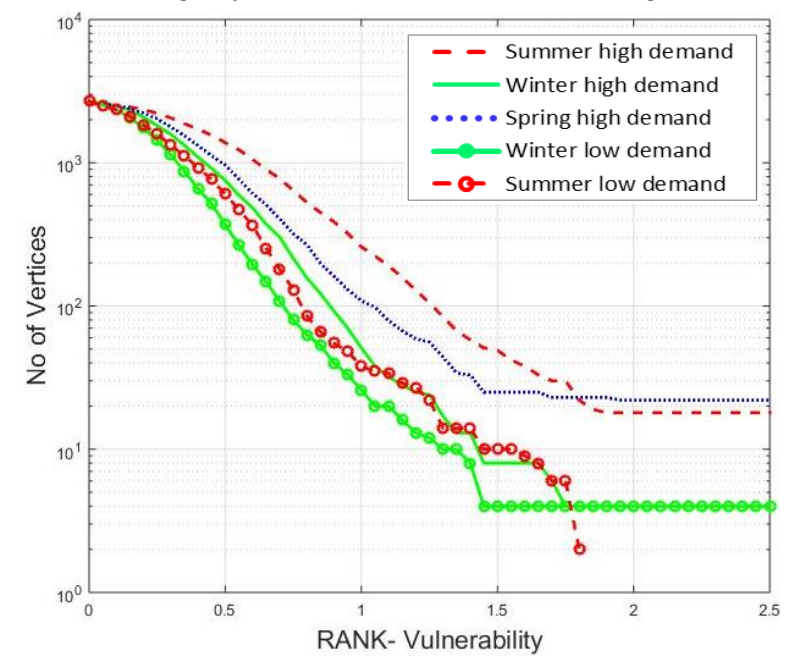

Fig. 3. RankV distributions for SW WI, 2016 base cases.

Modelling and data problems are the bane of the transition from theory to practice. Happily, here there were few such issues in mostly-excellent data, so valid conclusions can be drawn.

Two other explanations for the high RankVs for HSP and HS are more troubling. First, the operators may be counted on to respond in real-time to some contingencies in high-stress conditions. But failures in practices and procedures, including operator actions, are common triggers for cascading. The 8 Sept. 2011 blackout involved precisely such failures. Second, these high RankV situations may become the next unforeseen blackout.

Fig. 3 and similar distributions for the other three metrics are dramatic but difficult to work with. Table III, from vertical cuts through the distributions, is easier to handle.

For the SW WI system, RankV is the number of branches with vulnerability rank $\geq 1$, that is, with $n-1$ flows $\geq 100 \%$ of the branch rating. This amounts to 259 branches for HS $-10.7 \%$ of the branches in the system, which is a lot. For HS, 108 branches will have $n-1$ flows $\geq$ rating for at least two separate contingencies (DegreeV $\geq 2$ ).

Criticality is a less familiar concept. In HS, for each of 309 single contingencies, at least 1 monitored branch will have n-1 flows $\geq$ threshold $(100 \%$ of rating in this table). DegreeC $\geq 2$ means that for each of 89 single contingencies (in HS), n-1 flows on two or more monitored lines will exceed threshold.

Table III also includes an ad hoc judgment on how much the system is at risk to cascading. Demand and 
imports/exports (Table II) are considered to be first order stressors, and common practice to avoid maintenance in peak seasons is considered to be a second order stressor.

For the 2016 data, with two minor exceptions the four metrics are consistent with this ad hoc stress judgment. (The 2011 data will be discussed later.) Most values of the metrics differ in the first significant figure. The differentiation is quite clear.

TABLE III

SW WI stress metrics and ad hoc risk judgment

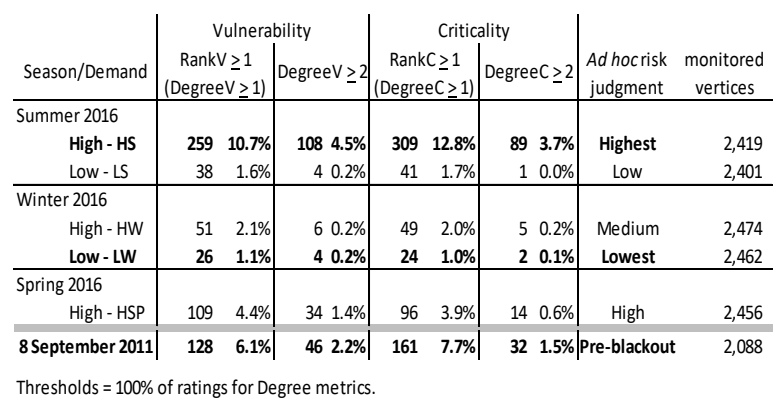

Planners and operators could identify the high vulnerability branches and look for ways to reduce it, as higher stress means higher risk of cascading. They might focus more on the highly critical branches: some outages could cause high n-1 loadings; a few of these may affect many branches. Or they could seek ways to make their system inherently less stressed.

\subsection{Pre-blackout metrics, 8 September 2011}

On 8 September 2011, a switching error tripped the $500 \mathrm{kV}$ Hassayampa - North Gila line. The line had tripped before without cascading, but on this day all the SW WI metrics showed high stress (Table III). The $500 \mathrm{kV}$ outage caused the two Coachella $230 \mathrm{kV} / 92 \mathrm{kV}$ transformers to overload and trip. Further cascading blacked out much of SW WI [33].

Metrics show where stress was highest: $7.5 \%$ of Imperial Irrigation District branches (Table IV, row 2) had RankV > 1, well above the SW WI average. Six, including the Coachella transformers, were vulnerable for two or more n-1 outages.

Cascading began in Arizona (row 1), with over one-third of the critical SW WI branches, including the initiating outage.

An area being stressed does not mean that cascading will occur or start there. But history is clear: cascading occurs when a system is stressed. Had the operators known, they could have reduced the stress. A switching error need not have cascaded.
TABLE IV

Pre-cascading metrics, seven SW WI areas and total, 8 September 2011

\begin{tabular}{r|rrrr|rrrr} 
& \multicolumn{4}{|c|}{ Vulnerability } & \multicolumn{5}{c}{ Criticality } \\
Branches & $\begin{array}{r}|c| \\
\text { RANKV } \geq 1\end{array}$ & DEGREEV $\geq 2$ & \multicolumn{2}{c}{ RANKC $\geq 1$} & DEGREEC $\geq 2$ \\
& $($ DegreeV $\geq 1)$ & & & (DegreeC $\geq 1)$ & & \\
\hline 1,542 & 39 & $2.5 \%$ & 25 & $1.6 \%$ & 73 & $4.7 \%$ & $\mathbf{7}$ & $0.5 \%$ \\
134 & $\mathbf{1 0}$ & $\mathbf{7 . 5 \%}$ & $\mathbf{6}$ & $\mathbf{4 . 5 \%}$ & 7 & $5.2 \%$ & 1 & $0.7 \%$ \\
272 & 0 & $0.0 \%$ & 0 & $0.0 \%$ & 1 & $0.4 \%$ & 1 & $0.4 \%$ \\
\hline 255 & 4 & $1.6 \%$ & 2 & $0.8 \%$ & 4 & $1.6 \%$ & 0 & $0.0 \%$ \\
506 & 6 & $1.2 \%$ & 2 & $0.4 \%$ & 14 & $2.8 \%$ & 0 & $0.0 \%$ \\
397 & 6 & $1.5 \%$ & 1 & $0.3 \%$ & 11 & $2.8 \%$ & 4 & $1.0 \%$ \\
\hline 1,057 & 62 & $5.9 \%$ & 28 & $2.6 \%$ & 75 & $7.1 \%$ & $\mathbf{1 5}$ & $1.4 \%$ \\
& & & & & & & & \\
$\mathbf{4 , 0 9 6}$ & $\mathbf{1 2 6}$ & $\mathbf{3 . 1 \%}$ & $\mathbf{6 3}$ & $\mathbf{1 . 5}$ & $\mathbf{1 8 3}$ & $\mathbf{4 . 5} \%$ & $\mathbf{2 7}$ & $\mathbf{0 . 7 \%}$
\end{tabular}

Notes: Here "branches" include non-outaged radial lines and transformers. A few branches are counted in more than one area.

\subsection{Inflections and Thresholds}

Change in demand can affect stress. Dealing with this effect takes in-depth knowledge of a system. WECC's 2016 cases embody careful, consistent modeling. In Fig. 4, stress goes up linearly with demand. At about 35,000 MW, the slope changes.

Another interpretation: at $62,700 \mathrm{MW}$ of demand, about 600 branches are loaded to or above 75 percent of their ratings for $n-1$ contingencies. Of these branches, 260 reach or exceed 100 percent of ratings, and 100 reach or exceed 125 percent of ratings. The system is highly stressed at this level of demand.

The pattern is the same for all thresholds. The value of the threshold affects the slope, but changes only a little the location of the inflection. Tipping points have been noted before [6] [34] [35].

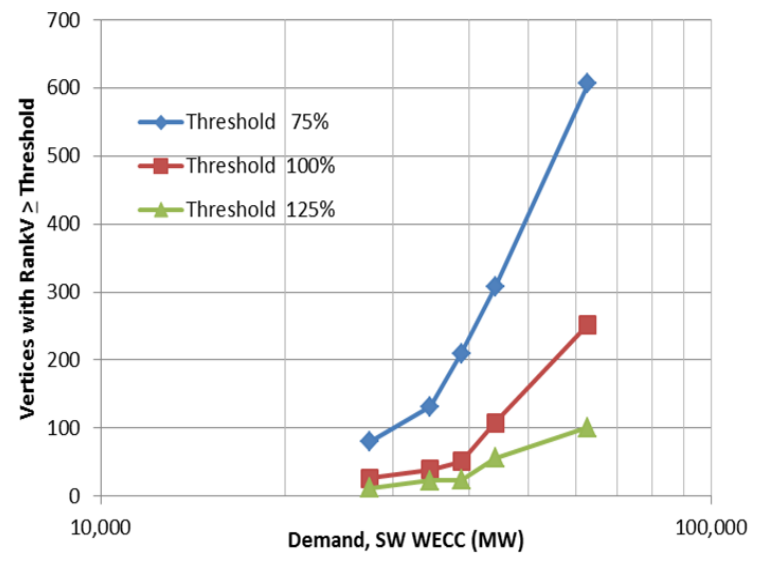

Fig. 4. Vulnerability rank as a function of demand, SW WI.

Setting thresholds too high or too low may give odd results. Choosing a reasonable threshold is like focusing a microscope, and is not difficult.

No claim is made that a branch will fail once flow reaches the threshold. Choosing thresholds for 
DegreeV and DegreeC is for making vertical cuts through the distributions, to create Tables III - V. The results are easier to work with than Fig 3 .

\subsection{Very large system studies}

For Table V, essentially all WI branches were outaged and monitored. Key areas (rows) are highlighted. In one, for $8 \%$ of its branches n-1 loadings met or exceeded the branch limits. This area and two others were the most vulnerable in the WI. They also had the highest fraction of branches that were overloaded by two or more single contingencies. A fourth high stress area also is noted.

One area had 455 RankC $\geq 1$ branches. If any of these branches had an outage, other branches would overload. For 339 very critical branches, two or more other branches would overload. One-third of the critical branches in the WI, and one- half of the highly critical ones, were in this area. Another area with high DegreeC is also found. An interesting conclusion is that stress-related problems can be different in nature for different areas.

\section{Adequacy and implications of metrics}

\subsection{Adequacy of stress metrics}

Three issues appear to have been overlooked in this work. First, cascading often includes non-linear effects like voltage collapse or transient instability. These usually result from high branch loading. It usually is not necessary to model these effects separately in computing the metrics.

In the studies above, the branch and path limits were determined from conductor thermal ratings, but reduced as needed for special voltage or transient stability concerns, etc. For each monitored branch the metrics measure how close n-1 flows are to these limits, including these adjustments. The method does not model all the phenomena associated with cascading. But there is a benefit to having a simple practical approximate technique if it can provide an effective warning signal in a real, large scale system. Our metrics can.

Linear DFAX analysis is an accepted way of approximating post-contingency MW flows on all of the other branches, for each contingency. But if the DFAX do not give accurate enough n-1 flows, a.c. contingency analysis, which is not too much slower, can be used in calculating metrics.

The second issue: cascading is an $\mathrm{n}-1-\mathrm{k}-\ldots$ event.
TABLE V

Western Interconnection metrics, Summer High load, 2016.

("Branches" include only monitored/outaged branches)

\begin{tabular}{|c|c|c|c|c|c|c|c|c|}
\hline \multirow{3}{*}{$\begin{array}{r}\text { Branches } \\
2,022\end{array}$} & \multicolumn{4}{|c|}{ Vulnerability } & \multicolumn{4}{|c|}{ Criticality } \\
\hline & \multicolumn{2}{|c|}{$\begin{array}{c}\text { RANKV }>1 \\
(D E G R E E V \geq 1)\end{array}$} & \multicolumn{2}{|c|}{ DEGREEV > 2} & \multicolumn{2}{|c|}{$\begin{array}{c}\text { RANKC }>1 \\
(\text { DEGREEC }>1)\end{array}$} & \multicolumn{2}{|c|}{ DEGREEC $>2$} \\
\hline & 53 & $2.6 \%$ & 26 & $1.3 \%$ & 154 & $7.6 \%$ & 48 & $2.4 \%$ \\
\hline 1,771 & 67 & $3.8 \%$ & 38 & $2.1 \%$ & 105 & $5.9 \%$ & 24 & $1.4 \%$ \\
\hline 3,042 & 107 & $3.5 \%$ & 61 & $2.0 \%$ & 135 & $4.4 \%$ & 57 & $1.9 \%$ \\
\hline 386 & 13 & $3.4 \%$ & 5 & $1.3 \%$ & 19 & $4.9 \%$ & 3 & $0.8 \%$ \\
\hline 312 & 16 & $5.1 \%$ & 8 & $2.6 \%$ & 20 & $6.4 \%$ & 6 & $1.9 \%$ \\
\hline 502 & 3 & $0.6 \%$ & 0 & $0.0 \%$ & 14 & $2.8 \%$ & 10 & $2.0 \%$ \\
\hline 327 & 8 & $2.4 \%$ & 4 & $1.2 \%$ & 8 & $2.4 \%$ & 2 & $0.6 \%$ \\
\hline 539 & 29 & $5.4 \%$ & 18 & $3.3 \%$ & 29 & $5.4 \%$ & 10 & $1.9 \%$ \\
\hline 263 & 9 & $3.4 \%$ & 8 & $3.0 \%$ & 28 & $10.6 \%$ & 6 & $2.3 \%$ \\
\hline 541 & 3 & $0.6 \%$ & 1 & $0.2 \%$ & 21 & $3.9 \%$ & 9 & $1.7 \%$ \\
\hline 746 & 16 & $2.1 \%$ & 2 & $0.3 \%$ & 17 & $2.3 \%$ & 0 & $0.0 \%$ \\
\hline 793 & 18 & $2.3 \%$ & 5 & $0.6 \%$ & 17 & $2.1 \%$ & 5 & $0.6 \%$ \\
\hline 4,973 & 122 & $2.5 \%$ & 49 & $1.0 \%$ & 455 & $9.1 \%$ & 339 & $6.8 \%$ \\
\hline 1,634 & 65 & $4.0 \%$ & 6 & $0.4 \%$ & 72 & $4.4 \%$ & 16 & $1.0 \%$ \\
\hline 4,471 & 149 & $3.3 \%$ & 71 & $1.6 \%$ & 211 & $4.7 \%$ & 58 & $1.3 \%$ \\
\hline 832 & 21 & $2.5 \%$ & 8 & $1.0 \%$ & 24 & $2.9 \%$ & 4 & $0.5 \%$ \\
\hline 504 & 42 & $8.3 \%$ & 18 & $3.6 \%$ & 72 & $14.3 \%$ & 25 & $5.0 \%$ \\
\hline 608 & 17 & $2.8 \%$ & 2 & $0.3 \%$ & 4 & $0.7 \%$ & 0 & $0.0 \%$ \\
\hline 1,281 & 102 & $8.0 \%$ & 54 & $4.2 \%$ & 98 & $7.7 \%$ & 45 & $3.5 \%$ \\
\hline 1,127 & 6 & $0.5 \%$ & 0 & $0.0 \%$ & 2 & $0.2 \%$ & 0 & $0.0 \%$ \\
\hline 49 & 2 & $4.1 \%$ & 1 & $2.0 \%$ & 2 & $4.1 \%$ & 0 & $0.0 \%$ \\
\hline 6,447 & 858 & $3.2 \%$ & 381 & $1.4 \%$ & 1,490 & $5.6 \%$ & 658 & $2.5 \%$ \\
\hline
\end{tabular}

Branches include lines and transformers.

Some Branches are included in more than one area.

One may ask, "How can the metrics, which come from n-1 analyses, measure the risk of cascading?"

The first and obvious part of the answer is that a necessary condition for cascading is that some first contingency cause a second contingency.

The metrics consider all possible first (n-1) contingencies. For each of these, the metrics evaluate the post-contingency flows on every branch. If all the metrics of vulnerability and criticality are low, the system is not stressed, and it is unlikely that n-1-1 cascading will occur. On the other hand, the more high indices there are, the more opportunities for an n-1-1 event, and for further cascading as well. In other words, the metrics for each n-1 state are indicative of the system risk beyond that $\mathrm{n}-1$ event.

High stress is necessary for cascading, we claim. It is not a sufficient condition. History reveals that the right failure also must occur in control and protection devices, or in practices and procedures.

The stress metrics do not attempt to forecast cascading. They quantify the risk of cascading.

A third issue: Our study did not model remedial action schemes (RAS). Most power flow programs 
could not do it then, but PowerWorld, which we used, could. One reviewer noted that WI RAS data usually is not given to researchers, and a "forgotten" RAS made the 2011 blackout worse. Modeling that RAS would not have changed our conclusions.

\subsection{Which metric is best?}

Each metric measures stress in a different way. None has been shown to be "best". Tests have not shown high correlations among them [32]. It is reasonable for the stress of a complex system to be multi-faceted, a vector, not a scalar. Combining the four metrics into a single metric would destroy information.

Medicine also uses a vector of four or five stress metrics, called vital signs. Measuring the metrics is simple, e.g., a thermometer scan of the forehead. It is more useful to know that "All vital signs are normal, except that the pulse is quite high" than that "The weighted sum of all the vital signs is a bit high."

\subsection{Two implications and a practical issue}

As demand and generation grow, transmission systems are expanded to increase transfer capability. Previous work showed that this expansion may increase the risk of cascading [6]. This important topic is beyond the scope of this paper.

Another important topic - how operators and planners may appropriately reduce stress - is also beyond the scope of this paper.

For real networks, the database is huge, but most DFAXes are very small. In this work, sparsity methods were used to store and compute only with DFAX absolute values above 0.001 .

\section{Summary and conclusions}

This paper presents significant advances on the oldest major unsolved technical problem in power systems, cascading blackouts.

Four metrics quantify stress, the risk of cascading. These metrics were computed for the Western Interconnection of North America (WI) using utility databases for different seasons and demands.

The same metrics were computed for a reconstruction of the pre-event state of a major cascading blackout. The metrics showed that the system was at high risk. They identified the areas where cascading developed, and the key branches involved, as being very vulnerable and critical.
Other interesting new results include:

- In a large system with low load growth, the number of large DFAX grew $32 \%$ in five years. More branches became tightly coupled.

- Stress varies reasonably and significantly in time and location, with differing needs for preventive action.

- Tipping points were observed in this and earlier studies using the metrics.

- 26,400 easy-to-do contingency analyses gave useful diagnoses of risk of cascading for all the WI, without running 350E+06 n-2 cases.

We conclude that the metrics are useful and practical measures of stress. The input data is a power flow base case or a real-time or stored state estimation snapshot. Proper use of these metrics will identify a system's susceptibility to cascading, and will pinpoint the most vulnerable and critical areas and facilities. The information can be used for planning, operations, and post-mortems.

\section{Acknowledgements}

This work was supported in part by a research contract from the Western Electricity Coordinating Council (WECC). This support is gratefully acknowledged. Of great value were the technical collaboration and data provided by the WECC, as acknowledged earlier. This highly unusual support contributed much to the success of this work.

\section{References}

[1] G. P. Loehr, "The 'good' blackout - the Northeast power failure of 9 November 1965," IEEE Power \& Energy Magazine, pp. 84-96, May/June 2017.

[2] U.S.-Canada Power System Outage Task Force, "Final Report on the August 14, 2003 Blackout in the United States and Canada," April 2004.

[3] Investigation Committee on the 28 September 2003 blackout in Italy, "Interim Report on the 28 September 2003 blackout in Italy," Union for the Coordination of Transmission of Electricity (UCTE), Brussels, 27 October 2003.

[4] Investigation Committee on the 28 September 2003 blackout in Italy, "Final Report on the 28 September 2003 blackout in Italy," UCTE, Brussels, April 2004.

[5] "American Electric Power Company Annual Report," 1965.

[6] H. M. Merrill and J. W. Feltes, "Cascading blackouts: stress, vulnerability, and criticality," 28 September 2016. [Online]. Available: http://www.MerrillEnergy.com. [Accessed 11 June 2020]. 
[7] ERCOT (Electric Reliability Council of Texas), "ERCOT Emergency Operation, December 21 - 23, 1989," ERCOT.

[8] ERCOT.com web site, "Event Summary -- Emergency Electric Curtailment Program - April 17, 2006," [Online]. Available: /ERCOT\%20April\%202006\%20LoadShed Report-April\%252017\%25202006.pdf.

[9] E. Vugrin, A. Castillo and C. Silva-Monroy, "Resilience metrics for the electric power system: a performance-based approach,," Sandia National Laboratories, Albuquerque, NM and Livermore, CA, 2017.

[10] B. Beasley and J. Greenwald, "Power system resilience: a primer," Bipartisan Policy Center, Washington, DC, February 2018.

[11] H. M. Merrill and J. W. Feltes, "Transmission icing: a physical risk with a physical hedge," Panel Session on Decision making under uncertainty, IEEE PES General Mtg., Montreal,WWW.MerrillEnergy.com, 2006.

[12] M. Elsberg, Blackouts: tomorrow will be too late, Penguin, 2012.

[13] "Standard TPL-001-4 - Transmission system planning performance requirements, in "Reliability standards for the bulk electric systems of North America"," NERC, Atlanta, 17 August 2016.

[14] F. C. Schweppe and E. Handschin, "Static state estimation in electric power systems," IEEE Proceedings, vol. 62, no. 7, pp. 972-982, 1974.

[15] L. Mili, Q. Qiu and A. G. Phadke, "Risk assessment of catastrophic failures in electric power systems," Int. J. Critical Infrastructures, vol. 1, no. 1, pp. 38-63, 2004.

[16] J. Chen, J. D. Thorp and I. Dobson, "Cascading dynamics and mitigation assessment in power system disturbances via a hidden failure model," Int. J. of Electrical Power and Energy Syst., vol. 27, no. 4, pp. 318-326, 2005.

[17] V. Bush, Pieces of the Action, New York: Morrow, 1970.

[18] F. C. Schweppe, "Power systems '2000': hierarchical control strategies," IEEE Spectrum, no. July, pp. 4247, 1978.

[19] IEEE Working Group on Understanding, Prediction, Mitigation and Restoration of Cascading Failures, "Benchmarking and validation of cascading failure analysis tools," IEEE Trans. Power Systems, vol. 31, no. 6, pp. 4887-4900, Nov 2016.

[20] M. E. J. Newman, "The structure and function of complex networks," SIAM Rev., vol. 45, no. 2, pp. 167-256, 2003.

[21] D. J. Watts, Six degrees: the science of a connected age, New York: W. W. Norton, 2003, pp. 104-107.

[22] S.-K. Chai and A. Sekar, "Graph theory application to deregulated power system," IEEE, 0-7803-6661 2001.

[23] Z. Wang, R. J. Thomas and A. Scaglione, "Generating random topology power grids," ECE Cornell University, Ithaca, NY.

[24] J.-C. Laprie, K. Kanoun and M. Kaâniche, "Modelling interdependencies between the electricity and information infrastructures," in SAFECOMP 2007, 2007.

[25] S. V. Buldyrev, R. Parshani, G. Paul, H. E. Stanley and S. Havlin, "Catastrophic cascade of failures in interdependent networks," Nature, vol. 464, pp. 10251028, 2010.

[26] K. Sun, "Complex networks theory: a new method of research in power grid," in IEEE/PES Trans. \& Dist. Conf. \& Exh., Dalian, China, 2005.

[27] I. Dobson, K. R. Wierzbicki, J. Kim and H. Ren, "Towards quantifying cascading blackout risk," in iREP Symposium - Bulk Power System Dynamics and Control, Charleston SC, USA, 2007.

[28] I. Dobson, "Where is the edge for cascading failure?: challenges and opportunities for quantifying blackout risk," in IEEE PES Gen Mtg, Tampa FL USA, 2007.

[29] N. Bhatt, S. Sarawgi, R. O'Keefe, P. Duggan, M. Koenig, M. Leschuk, S. Lee, K. Sun, V. Kolluri, S. Mandal, M. Peterson, D. Brotzman, S. Hedden, E. Litvinov, S. Maslennikov, X. Luo, E. Uzunovic, B. Fardanesh, L. Hopkins, A. Mander, K. Carman, M. Vaiman and M. Povolotskiy, "Assessing vulnerability to cascading outages," in Proc. IEEE/PES Power Systems Conference and Exposition, March 2009.

[30] H. M. Merrill, J. W. Feltes and D. J. Eckenfelder, Lessons learned in blackouts and their application in new planning criteria, CEIR Latin America Wkshp on Emergency State Op, Cuernavaca, Mexico, August 2009.

[31] H. M. Merrill and J. W. Feltes, "System Stress and Cascading Blackouts," in Advances in Electric Powser and Energy: Static State Estimation (M E El-Hawary, $E d)$, Hoboken, NJ, Wiley/IEEE Press, 2020.

[32] M. A. Hossain, M. Bodson and H. M. Merrill, "Evaluation of metrics of susceptibility to cascading blackouts," in Power and Energy Conf. at Illinois, Urbana, IL, 23-24 February 2017.

[33] FERC/ NERC, "Arizona- Southern California Outages on September 8, 2011, Causes and Recommendations," April 2012.

[34] D. P. Nedic, I. Dobson, D. S. Kirschen, B. A. Carreras and V. E. Lynch, "Criticality in a cascading failure blackout model," in 15th Power Systems Computation Conference, Liege, Belgium, 2005.

[35] H. Ren and D. Watts, "Early warning signals for critical transitions in power systems," Electric Power Systems Research, vol. 124, pp. 173-180, 2015. 